\title{
Characteristic Model-Based Adaptive Control for a Class of MIMO Uncertain Nonaffine Nonlinear Systems Governed by Differential Equations
}

\author{
Duo-Qing Sun $\mathbb{D}^{1,2}$ and Xiao-Ying $M a \mathbb{C}^{1}$ \\ ${ }^{1}$ Institute of Mathematics and Systems Science, Hebei Normal University of Science and Technology, Qinhuangdao, \\ Hebei 066004, China \\ ${ }^{2}$ Beijing Institute of Control Engineering, China Academy of Space Technology, Beijing 100190, China
}

Correspondence should be addressed to Duo-Qing Sun; sun_duoqing@126.com

Received 7 September 2019; Revised 1 December 2019; Accepted 23 December 2019; Published 26 February 2020

Academic Editor: Mojtaba Ahmadieh Khanesar

Copyright (c) 2020 Duo-Qing Sun and Xiao-Ying Ma. This is an open access article distributed under the Creative Commons Attribution License, which permits unrestricted use, distribution, and reproduction in any medium, provided the original work is properly cited.

\begin{abstract}
This paper addresses the difficulty of designing a controller for a class of multi-input multi-output uncertain nonaffine nonlinear systems governed by differential equations. We first derive the first-order characteristic model composed of a linear time-varying uncertain system for such nonaffine systems and then design an adaptive controller based on this first-order characteristic model for position tracking control. The designed controller exhibits a simple structure that can effectively avoid the controller singularity problem. The stability of the closed-loop system is analyzed using the Lyapunov method. The effectiveness of our proposed method is validated with a numerical example.
\end{abstract}

\section{Introduction}

The study of complex nonlinear systems governed by differential equations has attracted considerable attention [1-4]. It is worth noting that in the real world, there are many nonaffine nonlinear systems, such as certain flight control systems $[5,6]$, tank reactor system $[7,8]$, biochemical processes $[9,10]$, and carrier landing control systems for unmanned aerial vehicles [11]. The study of nonaffine systems is often much more difficult and complicated than that of affine ones because control inputs always appear implicitly in the nonlinear functions [7], and finding the explicit inversion of nonaffine functions is quite difficult, even when the existence of such nonlinear function inversion can be proved using the implicit function theorem $[5,9]$. On the other hand, in practical applications, uncertainties often arise owing to the presence of unknown parameter variations, modeling simplifications, unmodeled dynamics, and external disturbances, among others [12-14]. In certain practical situations, establishing accurate and detailed models of nonlinear systems may be difficult, costly, and time consuming. Therefore, the control design for uncertain nonaffine systems is an interesting yet challenging problem. Over the past decades, nonaffine nonlinear system control has received considerable attention, and many important results have been presented on the design of such controllers [5-12, 14, 15]. Owing to the universal approximation theorem, some adaptive control schemes based on fuzzy logic systems [16-22] and neural networks $[8,10,23-25]$ have been developed for uncertain nonaffine nonlinear systems. Nevertheless, these approaches typically incur heavy computational costs. Apart from these approaches, other adaptive control methods have also been considered for uncertain nonaffine-in-control nonlinear systems, such as adaptive dynamic surface control [7] and generalized PI control [26].

Recently, a majority of representative adaptive control schemes focuses on the continuous-time domain for nonaffine nonlinear systems described by differential equations. However, in engineering implementations, computers are 
usually utilized to produce digital control signals, which require controllers to be designed in discrete time [27].

The above discussion motivates us to use a novel method, called characteristic modeling proposed by $\mathrm{Wu}$ [28-35], to solve the control design problem for uncertain nonaffine systems. The key idea of the characteristic modeling is to use a lower-order discrete time-varying linear system to express an original continuous system equivalently based on both the dynamic characteristics of controlled plants and performance specifications. This discrete system is called the "characteristic model of the original system." It is worth mentioning that the characteristic modeling differs from the conventional model reduction methods in that all information of the original dynamic model, including uncertainties and nonlinearities, is compressed/integrated into the time-varying coefficients of the characteristic model instead of truncating parts of the plant model [28, 32,33]. The timevarying coefficients of the characteristic model are referred to as the characteristic parameters $[33,35,36]$, and the characteristic parameters are identified online adaptively $[31,33,35,37]$. The characteristic model-based adaptive control might be a fresh perspective in the field of adaptive control: creating simplicity out of complexity [31], and it has already been applied successfully to more than 400 engineering systems including spacecraft, e.g., the reentry control of a manned spaceship [28, 29, 32], the control of servo systems with backlash and friction [30], and the control for the swing arm in a Fourier transform spectrometer [38].

However, in the existing literature, the main focus is on the second-order characteristic model; the first-order model has received scarce attention for related results on controller design based on the characteristic model, and few studies have reported on the control method for nonaffine nonlinear systems based on the characteristic model. In the controller design method based on the second-order characteristic model, almost all the existing control methods belong to the indirect method in adaptive control. In other words, the model parameters are identified by the input and output of the system, and then the control law is designed. For the second-order characteristic model, the most successful application in practical engineering is the characteristic modelbased golden section adaptive control method, and it must also be combined with the integral controller and the differential controller to improve the control performance $[30,33,35]$. This makes it very difficult to analyze the stability of the control system. Stability analysis, especially for MIMO systems, remains a challenging problem for adaptive control based on the second-order characteristic model despite its practical successes. Compared with the secondorder characteristic model, the first-order model has the advantages of simpler form, fewer identified parameters, and more convenient applicability for practical systems.

In this paper, inspired by $[28,39]$, we derive the firstorder characteristic model for multi-input multi-output (MIMO) uncertain nonaffine nonlinear systems. Then, we develop a characteristic model-based adaptive control law for the position tracking problem. By constructing a suitable Lyapunov functional, we investigate the stability of the closed-loop system involving the first-order characteristic model-based control law.

Compared with the existing results, the primary contributions and novelties of our study can be highlighted as follows:

(1) The first-order characteristic model composed of a linear time-varying uncertain system is derived for a class of MIMO uncertain nonaffine nonlinear systems governed by differential equations. To the best of the authors' knowledge, first-order characteristic models have only been presented by $\mathrm{Xu}$ and $\mathrm{Hu}$ [39] and Sun et al. [40]. We note that SISO systems are only considered in $[39,40]$ and linear systems are only considered in [40].

(2) An adaptive controller based on this first-order characteristic model for position tracking control (see formula (22)) is designed by adaptively estimating the time-varying coefficients of the characteristic model (see formula (23)). In the existing literature, no results have been reported on the adaptive control design based on the first-order characteristic model for our considered class of nonaffine multivariable systems.

(3) The ranges of controller parameters, obtained (see Lemma 1) by utilizing some mathematical technique, guarantee the stability of the closed-loop system (see Theorem 2). In [39], no intervals of controller parameters are presented.

(4) The designed controller exhibits a simple structure and can effectively avoid possible controller singularity problems.

The paper is organized as follows. The problem formulation and the characteristic model are given in Section 2. Then, we present the design of the adaptive controller based on the characteristic model and the stability analysis by using the Lyapunov theory in Section 3. The effectiveness of the proposed method is illustrated by a numerical example in Section 4. Section 5 concludes this paper.

\section{Problem Formulation and Characteristic Modeling}

The following notation is used in the paper. $\|x\|$ denotes the Euclidean norm for a vector $x$. $\|A\|$ denotes the Frobenius norm for a matrix $A$. $R^{n}$ denotes the $n$-dimensional Euclidean space. $I$ is the $n$-dimensional identity matrix. Let $T_{s}>0$ be the sampling period.

Consider a class of MIMO uncertain nonaffine nonlinear systems:

$$
\dot{x}=f(x, u),
$$

where $x=\left[x_{1}, \ldots, x_{n}\right]^{\mathrm{T}} \in R^{n}$ and $u=\left[u_{1}, \ldots, u_{n}\right]^{\mathrm{T}} \in R^{n}$ represent the state (output) and the input vector of the controlled plant, respectively. $f(x, u)=\left[f_{1}(x, u), \ldots\right.$, $\left.f_{n}(x, u)\right]^{\mathrm{T}}$ is an unknown smooth nonlinear function vector. 
Let $r_{i} \in(0,1)$ for $i=1,2, \ldots, n$. For Jacobian matrix $\partial f(x, u) / \partial u, u$ in the $i$ th row is replaced by $r_{i} u$, and the resulting matrix is denoted as $f_{u}(x, \cdot)$. We suppose that system (1) satisfies the following assumptions.

Assumption 1. $f_{u}(x, \cdot)$ is nonsingular.

Assumption 2. Each $f_{i}(x, 0)$ and $\left\|f_{u}(x, \cdot)^{-1}\right\|$ are bounded.

The control objective is to design the sampled data controller $u_{i}(t)=u_{i}\left(k T_{s}\right), k T_{s} \leq t \leq(k+1) T_{s}$, such that the output $x_{i}(t)$ of the controlled system (1) tracks the preset value $x_{i r}, i=1,2, \ldots, n$.

We first define the following set:

$$
\Omega_{i}=\left\{x_{i} \in R|| x_{i} \mid \leq \eta_{i}\right\}, \quad i=1,2, \ldots, n,
$$

where $\eta_{i}$ is a small positive constant.

Denote $x_{i, k}=x_{i}\left(k T_{s}\right)$ and $u_{i, k}=u_{i}\left(k T_{s}\right)$ for $i=1$, $2, \ldots, n$. Let

$$
y_{i, k}=x_{i . k}+D_{i} d_{i}, \quad i=1,2, \ldots, n,
$$

where $d_{i}>\eta_{i}$ is a constant; $D_{i}=1$ if $x_{i, k} \in \Omega_{i}$ and $D_{i}=0$ if $x_{i, k} \notin \Omega_{i}$; we refer to $y_{i, k}$ as the unified variable. From (3), we can see that unified variables $y_{i, k}$ are not close to zero.
Theorem 1. Given an MIMO nonaffine nonlinear system (1) satisfying Assumption 1, if position keeping or position tracking control is desired, then its characteristic model can be described by the following system of first-order difference equations:

$$
y_{i, k+1}=\theta_{i, k} y_{i, k}+T_{s} u_{i, k}, \quad i=1,2, \ldots, n,
$$

where $\theta_{i, k}$ is the so-called characteristic parameter as follows:

$$
\theta_{i, k}=1+\varepsilon_{i, k}, \quad \varepsilon_{i, k}=\frac{g_{i, k}}{y_{i, k}},
$$

where $g_{i, k}$ is the ith component of the vector $\int_{k T_{s}}^{(k+1) T_{s}}$ $\left[\left(I-f_{u}(x, \cdot)^{-1}\right) \dot{x}+f_{u}(x, \cdot)^{-1} f(x, 0)\right] d s$.

Proof. For a fixed $i(i=1, \ldots, n)$, the following equation can be obtained based on the differential mean value theorem:

$$
\dot{x}_{i}=f_{i}(x, 0)+\bar{f}_{i}\left(x, \bar{u}_{i}\right) u, \quad i=1,2, \ldots, n,
$$

where $\bar{u}_{i}=r_{i} u, 0<r_{i}<1$,

$$
\bar{f}_{i}\left(x, \bar{u}_{i}\right)=\left[\bar{f}_{i 1}\left(x, \bar{u}_{i}\right), \bar{f}_{i 2}\left(x, \bar{u}_{i}\right), \ldots, \bar{f}_{i n}\left(x, \bar{u}_{i}\right)\right]=\left.\frac{\partial f_{i}(x, u)}{\partial u}\right|_{u=\bar{u}_{i}}, \quad \bar{f}_{i l}\left(x, \bar{u}_{i}\right)=\left.\frac{\partial f_{i}(x, u)}{\partial u_{l}}\right|_{u=\bar{u}_{i}} .
$$

Therefore, we have

$$
\dot{x}=f(x, 0)+\bar{f} u,
$$

where $\quad \bar{f}=f_{u}(x, \cdot)=\left[\bar{f}_{1}\left(x, \bar{u}_{1}\right)^{\mathrm{T}}, \bar{f}_{2}\left(x, \bar{u}_{2}\right)^{\mathrm{T}}, \ldots, \bar{f}_{n}(x\right.$, $\left.\left.\bar{u}_{n}\right)^{\mathrm{T}}\right]^{\mathrm{T}}$.

According to Assumption 1 , the inverse function $\bar{f}^{-1}$ must exist. Thus, multiplying both sides of (8) with $\bar{f}^{-1}$ yields

$$
\bar{f}^{-1} \dot{x}=\bar{f}^{-1} f(x, 0)+u \text {. }
$$

Therefore, we have

$$
\dot{x}=\left(I-\bar{f}^{-1}\right) \dot{x}+\bar{f}^{-1} f(x, 0)+u .
$$

Integrating both sides of the above equation yields

$$
x(t)=x(0)+\int_{0}^{t}\left[\left(I-\bar{f}^{-1}\right) \dot{x}+\bar{f}^{-1} f(x, 0)\right] \mathrm{d} s+\int_{0}^{t} u(s) \mathrm{d} s .
$$

Now set $t=(k+1) T_{s}$; then, (11) can be rewritten as

$$
\begin{aligned}
x\left((k+1) T_{s}\right)= & x(0)+\int_{0}^{(k+1) T_{s}}\left[\left(I-\bar{f}^{-1}\right) \dot{x}\right. \\
& \left.+\bar{f}^{-1} f(x, 0)\right] \mathrm{d} s+\int_{0}^{(k+1) T_{s}} u(s) \mathrm{d} s .
\end{aligned}
$$

Setting $t=k T_{s}$, (11) can also be rewritten as

$$
\begin{aligned}
x\left(k T_{s}\right)= & x(0)+\int_{0}^{k T_{s}}\left[\left(I-\bar{f}^{-1}\right) \dot{x}+\bar{f}^{-1} f(x, 0)\right] \mathrm{d} s \\
& +\int_{0}^{k T_{s}} u(s) \mathrm{d} s .
\end{aligned}
$$

Subtracting (13) from (12) yields

$$
\begin{aligned}
x\left((k+1) T_{s}\right) & =x\left(k T_{s}\right)+\int_{k T_{s}}^{(k+1) T_{s}}\left[\left(I-\bar{f}^{-1}\right) \dot{x}+\bar{f}^{-1} f(x, 0)\right] \mathrm{d} s+\int_{k T_{s}}^{(k+1) T_{s}} u(s) \mathrm{d} s \\
& =x\left(k T_{s}\right)+\int_{k T_{s}}^{(k+1) T_{s}}\left[\left(I-\bar{f}^{-1}\right) \dot{x}+\bar{f}^{-1} f(x, 0)\right] \mathrm{d} s+T_{s} u\left(k T_{s}\right) .
\end{aligned}
$$


Denote

$$
\begin{aligned}
g_{k} & =\left(g_{1, k}, g_{2, k}, \ldots, g_{n, k}\right)^{\mathrm{T}} \\
& =\int_{k T_{s}}^{(k+1) T_{s}}\left[\left(I-\bar{f}^{-1}\right) \dot{x}+\bar{f}^{-1} f(x, 0)\right] \mathrm{d} s, \\
u_{k} & =\left(u_{1, k}, u_{2, k}, \ldots, u_{n, k}\right)^{\mathrm{T}}=u\left(k T_{s}\right) .
\end{aligned}
$$

Then, we have $x\left((k+1) T_{s}\right)=x\left(k T_{s}\right)+g_{k}+u_{k}$, or equivalently,

$$
x_{i}\left((k+1) T_{s}\right)=x_{i}\left(k T_{s}\right)+g_{i, k}+T_{s} u_{i, k}, \quad i=1,2, \ldots, n .
$$

Next, we analyze two cases individually.

Case 1. If $x_{i}(k) \notin \Omega_{i}$, then the $i$ th output from the controlled object is far away from zero. Therefore, (17) can be rewritten as

$$
x_{i}\left((k+1) T_{s}\right)=\left[1+\frac{g_{i, k}}{x_{i}\left(k T_{s}\right)}\right] x_{i}\left(k T_{s}\right)+T_{s} u_{i, k} .
$$

Case 2. If $x_{i}(k) \in \Omega_{i}$, then the $i$ th output from the controlled object is close to zero. In this case, (17) can be rewritten as

$$
\begin{aligned}
x_{i}\left((k+1) T_{s}\right)+d_{i} & =x_{i}\left(k T_{s}\right)+d_{i}+g_{i, k}+T_{s} u_{i, k} \\
& =\left[1+\frac{g_{i, k}}{x_{i}\left(k T_{s}\right)+d_{i}}\right]\left(x_{i}\left(k T_{s}\right)+d_{i}\right)+T_{s} u_{i, k} .
\end{aligned}
$$

Combining Cases 1 and 2 from (3), we can obtain the characteristic model described by (4).

Remark 1. From Assumption 2, we can derive the range of the characteristic parameter $\theta_{i, k}$. In fact, from Assumption 2, there exist positive constants $M_{f}$ and $M_{u}$ such that $\|f(x, 0)\| \leq M_{f}$ and $\left\|\bar{f}^{-1}\right\|=\left\|f_{u}(x, \cdot)^{-1}\right\| \leq M_{u}$. We note that $\dot{x}$ is continuous. Thus, $\dot{x}$ is bounded on each closed interval $\left[k T_{s},(k+1) T_{s}\right]$. That is, there exist positive constants $M_{x k}$ such that $\|\dot{x}\| \leq M_{x k}$ on $\left[k T_{s},(k+1) T_{s}\right]$. Further, $\|\dot{x}\| \leq M_{x}=$ Supremum $_{k}\left\{M_{x k}\right\}$. This is not restrictive, and the supremum of $\left\{M_{x k}\right\}$ exists in practical engineering systems [41, 42]. Therefore, $\left\|\left(I-f^{-1}\right) \dot{x}+f^{-1} f(x, 0)\right\| \leq$ $\left\|\left(I-\bar{f}^{-1}\right) \dot{x}\right\|+\left\|\bar{f}^{-1} f(x, 0)\right\| \leq\left\|\left(I-\bar{f}^{-1}\right)\right\| \cdot\|\dot{x}\|+\| \bar{f}^{-1} f(x$, $0)\|\leq\| I\|\cdot\| \dot{x}\|+\| \bar{f}^{-1}\|\cdot\| \dot{x}\|+\| \bar{f}^{-1}\|\cdot\| f(x, 0)\|\leq\| I \| \cdot M_{x}+$ $M_{u} \cdot M_{x}+M_{u} \cdot M_{f}$.

We denote $G=\|I\| \cdot M_{x}+M_{u} \cdot M_{x}+M_{u} \cdot M_{f}$. Thus, we have $\left|\bar{g}_{i, k}\right| \leq G$, where $\bar{g}_{i, k}$ is the $i$ th component of the vector $\left(I-\bar{f}^{-1}\right) \dot{x}+\bar{f}^{-1} f(x, 0)$; then, $\left|g_{i, k}\right| \leq G T_{s}$, and we obtain $\left|\varepsilon_{i, k}\right|<\varepsilon_{i}$, where $\varepsilon_{i}=G T_{s} / \eta_{i}$ if $x_{i}\left(k T_{s}\right) \notin \Omega_{i}$ and $\varepsilon_{i}=$ $G T_{s} /\left(d_{i}-\eta_{i}\right)$ if $x_{i}\left(k T_{s}\right) \in \Omega_{i}$. From (5), we get

$$
1-\varepsilon_{i}=\delta_{i 1}<\theta_{i, k}<\delta_{i 2}=1+\varepsilon_{i} \text {. }
$$

\section{Controller Design and Stability Analysis}

We define the tracking errors as $e_{i, k}=x_{i, k}-x_{i r}$ for $i=1,2, \ldots, n$. The reference tracking signal of the unified variable $y_{i, k}$ can be expressed by $y_{i r}=x_{i r}+D_{i} d_{i}$. Note that the tracking errors of the unified variables equal the ones of the original object, which can be seen from

$$
\begin{aligned}
y_{i, k}-y_{i r} & =x_{i, k}+D_{i} d_{i}-\left(x_{i r}+D_{i} d_{i}\right) \\
& =x_{i, k}-x_{i r}=e_{i, k} .
\end{aligned}
$$

We present the basic approach to obtain an adaptive controller that achieves our control objective. If the function $f$ is known, then each $\theta_{i, k}$ is also known. The control laws $u_{i, k}^{*}=\left(-\theta_{i, k} y_{i, k}+a_{i 0} e_{i, k}+y_{i r}\right) / T_{s}(i=1,2, \ldots, n)$ applied to (4) result in $y_{i, k+1}=a_{i 0} e_{i, k}+y_{i r}$, that is, $e_{i, k+1}-a_{i 0} e_{i, k}=0$. Notice that if one chooses $a_{i 0}$ such that $\left|a_{i 0}\right|<1$, where $i=1,2, \ldots, n$, then the roots of the polynomials $H\left(q_{i}\right)=$ $q_{i}-a_{i 0}$ related to the characteristic equation of $e_{i, k+1}-a_{i 0} e_{i, k}=0$ are inside unit circles, which implies that $\lim _{k \longrightarrow+\infty} e_{i, k}=0$. This is main objective of control. Because $f$ is unknown, the optimal control $u_{i, k}^{*}$ cannot be implemented. Thus, our purpose is to design an adaptive controller to approximate this optimal control.

Based on the above ideas, the adaptive controller is designed as

$$
u_{i, k}=\frac{1}{T_{s}}\left(-\widehat{\theta}_{i, k} y_{i, k}+a_{i 0} e_{i, k}+y_{i r}\right), \quad i=1,2, \ldots, n,
$$

where $a_{i 0} \in(0,1)$ and $\widehat{\theta}_{i, k}$ denotes the estimation of the parameter $\theta_{i, k}$.

Note that $a_{i 0} \in(-1,0]$ is not considered for the convenience of theoretical analysis.

The adaptive law of parameter $\theta_{i, k}$ is designed as

$$
\widehat{\theta}_{i, k}=\frac{m_{i} \widehat{\theta}_{i, k-1}+\lambda_{i} a_{i 0} y_{i, k} e_{i, k}}{1+\lambda_{i} \mu_{i} y_{i, k}^{2}}
$$

where $\lambda_{i}>0, \mu_{i}>0$, and $0<m_{i}<1$ represent design constants.

The adaptive controller (22) includes estimated parameters $\widehat{\theta}_{i, k}$, and the performance of asymptotic tracking with zero error or near zero can be obtained by adjusting the design parameters $\lambda_{i}>0, \mu_{i}>0$, and $0<m_{i}<1$.

Let $\varphi_{i, k}=\theta_{i, k}-\hat{\theta}_{i, k}$ be the parameter estimation error. The error equation of the closed-loop system can be expressed by substituting (22) into (4) as follows:

$$
e_{i, k+1}=\left(\theta_{i, k}-\widehat{\theta}_{i, k}\right) y_{i, k}+a_{i 0} e_{i, k}=\varphi_{i, k} y_{i, k}+a_{i 0} e_{i, k}
$$

From (23), we have

$$
\widehat{\theta}_{i, k}-m_{i} \widehat{\theta}_{i, k-1}=\lambda_{i}\left(a_{i 0} y_{i, k} e_{i, k}-\mu_{i} y_{i, k}^{2} \widehat{\theta}_{i, k}\right) .
$$

Thus, as the basis for preparing the stability analysis of the closed-loop system, the relation between the parameter estimation errors at two adjacent instants can be obtained as follows: 


$$
\begin{aligned}
\varphi_{i, k} & =\theta_{i, k}-\widehat{\theta}_{i, k}+m_{i} \varphi_{i, k-1}-m_{i} \varphi_{i, k-1} \\
& =m_{i} \varphi_{i, k-1}+\theta_{i, k}-\widehat{\theta}_{i, k}-m_{i}\left(\theta_{i, k-1}-\widehat{\theta}_{i, k-1}\right) \\
& =m_{i} \varphi_{i, k-1}+\theta_{i, k}-m_{i} \theta_{i, k-1}-\left(\widehat{\theta}_{i, k}-m_{i} \widehat{\theta}_{i, k-1}\right) \\
& =m_{i} \varphi_{i, k-1}+\theta_{i, k}-m_{i} \theta_{i, k-1}-\lambda_{i}\left(a_{i 0} y_{i, k} e_{i, k}-\mu_{i} y_{i, k}^{2} \widehat{\theta}_{i, k}\right) \\
& =m_{i} \varphi_{i, k-1}+\theta_{i, k}-m_{i} \theta_{i, k-1}-\lambda_{i} a_{i 0} y_{i, k} e_{i, k}+\lambda_{i} \mu_{i} y_{i, k}^{2}\left(\theta_{i, k}-\varphi_{i, k}\right) \\
& =m_{i} \varphi_{i, k-1}+w_{i, k}
\end{aligned}
$$

where

$$
\begin{aligned}
w_{i, k}= & \left(\theta_{i, k}-m_{i} \theta_{i, k-1}\right)-\lambda_{i} a_{i 0} y_{i, k} e_{i, k} \\
& +\lambda_{i} \mu_{i} \theta_{i, k} y_{i, k}^{2}-\lambda_{i} \mu_{i} \varphi_{i, k} y_{i, k}^{2} .
\end{aligned}
$$

For subsequent use, we denote

$$
\begin{aligned}
& \lambda_{i 1}=\frac{m_{i}^{2} \Delta_{i}-m_{i}^{2} \sqrt{\Delta_{i}^{2}-c_{i}^{2}}}{c_{i}^{2}}, \\
& \lambda_{i 2}=\frac{m_{i}^{2} \Delta_{i}+m_{i}^{2} \sqrt{\Delta_{i}^{2}-c_{i}^{2}}}{c_{i}^{2}},
\end{aligned}
$$

where $\quad \Delta_{i}=-a_{i 0} c_{i}+\left(1-a_{i 0}^{2}\right) \mu_{i}, \quad c_{i}=\mu_{i} \delta_{i 2}-a_{i 0} ; \quad i=1$, $2, \ldots, n$. Then, we have the following lemma.

Lemma 1. Let $\mu_{i}$ satisfy

$$
\frac{a_{i 0}}{\delta_{i 2}}<\mu_{i}<\frac{a_{i 0}}{\delta_{i 2}-1+a_{i 0}}, \quad i=1,2, \ldots, n .
$$

Then, $\mu_{i}, \lambda_{i 1}, \lambda_{i 2}>0$, and $\lambda_{i 1}<\lambda_{i 2}$.

Proof. For the sake of simplicity, the subscript $i$ for all variables is omitted in this proof. For example, $a_{0}$ written in this proof actually represents $a_{i 0}$.

We first need to prove that (30) is well defined. It should be noted that $\delta_{2}>\delta_{2}-1+a_{0}=1+\varepsilon-1+a_{0}>0$. Therefore, (30) is well defined and clearly $\mu>0$.

To prove that $\lambda_{1}$ and $\lambda_{2}$ are also well defined, we need to first prove that $\Delta^{2}-c^{2}>0$. To do this, let us consider the following equality:

$$
\begin{aligned}
\Delta-c & =-a_{0} c+\left(1-a_{0}^{2}\right) \mu-c=-\left(1+a_{0}\right) c+\left(1-a_{0}^{2}\right) \mu \\
& =-\left(1+a_{0}\right)\left[\left(\delta_{2}-1+a_{0}\right) \mu-a_{0}\right] .
\end{aligned}
$$

Since $\mu<a_{0} /\left(\delta_{2}-1+a_{0}\right)$, we have $\Delta>c$. Considering that $\mu>a_{0} / \delta_{2}$, we obtain $c=\mu \delta_{2}-a_{0}>0$. Thus, we further have $\Delta>0$, and hence $\Delta^{2}-c^{2}>0$. Therefore, both $\lambda_{1}$ and $\lambda_{2}$ are well defined. It is clear that $\lambda_{1}, \lambda_{2}>0$ and $\lambda_{1}<\lambda_{2}$.
We set $T_{i}=\min \left\{\eta_{i},\left(d_{i}-\eta_{i}\right)\right\}, i=1,2, \ldots, n ; T_{\min }=$ $\min _{1 \leq i \leq n}\left\{T_{i} / G_{i}\right\}$. We require the following lemma to summarize the performance of our proposed control strategy.

Lemma 2 (see [43], Lemma 6). Let $x, y \in R$. Then,

$$
2 x y \leq \varepsilon x^{2}+\frac{1}{\varepsilon} y^{2},
$$

for any $\varepsilon>0$.

Theorem 2. Consider an MIMO nonaffine nonlinear system (1) satisfying Assumptions 1 and 2. If the adaptive tracking controller is designed as (22) with update law (23), the sampling period $T_{s}<T_{\min }$, and controller parameters $\mu_{i} \in\left(a_{i 0} / \delta_{i 1}, a_{i 0} /\left(\delta_{i 2}-1+a_{i 0}\right)\right), \lambda_{i} \in\left(\lambda_{i 1}, \lambda_{i 2}\right)(i=1,2, \ldots$, $n)$, then the following properties are guaranteed:

(1) The tracking errors $e_{i, k}(i=1,2, \ldots, n)$ and each parameter estimation error $\varphi_{i, k}$ are both bounded.

(2) The tracking errors $e_{i, k}(i=1,2, \ldots, n)$ converge to the neighborhoods of the origin.

Proof. First, we note that from (20), $\varepsilon_{i}<1$ when the sampling period $T_{s}<T_{\min }$. Thus, $\delta_{i 1}=1-\varepsilon_{i}>0$. Since $\mu_{i}>a_{i 0} / \delta_{i 1}$, it is easy to see that $\mu_{i}>a_{i 0} / \delta_{i 2}$. From Lemma 1 , there exist real numbers $\lambda_{i}$ such that $\lambda_{i} \in\left(\lambda_{i 1}, \lambda_{i 2}\right)$ for $i=1,2, \ldots, n$. Now, consider the Lyapunov function given by

$$
V_{i, k}=e_{i, k+1}^{2}+\varphi_{i, k}^{2}
$$

Part 1. Calculating the difference of the Lyapunov function (33).

By utilizing (24), we can derive the first difference of the Lyapunov function (33) as

$$
\begin{aligned}
\Delta V_{i, k} & =V_{i, k}-V_{i, k-1} \\
& =e_{i, k+1}^{2}-e_{i, k}^{2}+\varphi_{i, k}^{2}-\varphi_{i, k-1}^{2} \\
& =\left(\varphi_{i, k} y_{i, k}+a_{i 0} e_{i, k}\right)^{2}-e_{i, k}^{2}+\left(\varphi_{i, k}^{2}-\varphi_{i, k-1}^{2}\right) \\
& =a_{i 0}^{2} e_{i, k}^{2}+\varphi_{i, k}^{2} y_{i, k}^{2}+2 a_{i 0} \varphi_{i, k} y_{i, k} e_{i, k}-e_{i, k}^{2}+\left(\varphi_{i, k}^{2}-\varphi_{i, k-1}^{2}\right) .
\end{aligned}
$$

Using (26), the last term $-\varphi_{i, k-1}^{2}$ in (34) can be written as

$$
-\varphi_{i, k-1}^{2}=-\left(\frac{\varphi_{i, k}-w_{i, k}}{m_{i}}\right)^{2}=-\frac{1}{m_{i}^{2}} \varphi_{i, k}^{2}-\frac{w_{i, k}^{2}}{m_{i}^{2}}+\frac{2 \varphi_{i, k} w_{i, k}}{m_{i}^{2}} .
$$

Now, substituting (27) into $\varphi_{i, k} w_{i, k}$ in the last term of (35) and employing $y_{i, k}=e_{i, k}+y_{i r}$, we then have 


$$
\begin{aligned}
\varphi_{i, k} w_{i, k} & =\varphi_{i, k}\left[\left(\theta_{i, k}-m_{i} \theta_{i, k-1}\right)-\lambda_{i} a_{i 0} y_{i, k} e_{i, k}+\lambda_{i} \mu_{i} \theta_{i, k} y_{i, k}^{2}-\lambda_{i} \mu_{i} \varphi_{i, k} y_{i, k}^{2}\right] \\
& =\left(\theta_{i, k}-m_{i} \theta_{i, k-1}\right) \varphi_{i, k}-\lambda_{i} a_{i 0} \varphi_{i, k} y_{i, k} e_{i, k}+\lambda_{i} \mu_{i} \theta_{i, k} y_{i, k}^{2} \varphi_{i, k}-\lambda_{i} \mu_{i} \varphi_{i, k}^{2} y_{i, k}^{2} \\
& =\left(\theta_{i, k}-m_{i} \theta_{i, k-1}\right) \varphi_{i, k}-\lambda_{i} a_{i 0} \varphi_{i, k} y_{i, k} e_{i, k}+\lambda_{i} \mu_{i} \theta_{i, k} \varphi_{i, k} y_{i, k}\left(e_{i, k}+y_{i r}\right)-\lambda_{i} \mu_{i} \varphi_{i, k}^{2} y_{i, k}^{2} \\
& =-\lambda_{i} \mu_{i} \varphi_{i, k}^{2} y_{i, k}^{2}+\left(\theta_{i, k}-m_{i} \theta_{i, k-1}\right) \varphi_{i, k}+\lambda_{i} \varphi_{i, k} y_{i, k} e_{i, k}\left(-a_{i 0}+\mu_{i} \theta_{i, k}\right)+\lambda_{i} \mu_{i} \theta_{i, k} \varphi_{i, k} y_{i, k} y_{i r}
\end{aligned}
$$

Substituting (36) into (35) and then into (34) yields

$$
\begin{aligned}
\begin{aligned}
& \Delta V_{i, k}=\left(-1+a_{i 0}^{2}\right) e_{i, k}^{2}+\varphi_{i, k}^{2} y_{i, k}^{2}+2 a_{i 0} \varphi_{i, k} y_{i, k} e_{i, k}+\varphi_{i, k}^{2}-\frac{1}{m_{i}^{2}} \varphi_{i, k}^{2}-\frac{w_{i, k}^{2}}{m_{i}^{2}} \\
&+\frac{2}{m_{i}^{2}}\left[-\lambda_{i} \mu_{i} \varphi_{i, k}^{2} y_{i, k}^{2}+\left(\theta_{i, k}-m_{i} \theta_{i, k-1}\right) \varphi_{i, k}+\lambda_{i} \varphi_{i, k} y_{i, k} e_{i, k}\left(-a_{i 0}+\mu_{i} \theta_{i, k}\right)+\lambda_{i} \mu_{i} \theta_{i, k} \varphi_{i, k} y_{i, k} y_{i r}\right] \\
&=\left(-1+a_{i 0}^{2}\right) e_{i, k}^{2}+\left(-\frac{1}{m_{i}^{2}}+1\right) \varphi_{i, k}^{2}-\frac{1}{m_{i}^{2}} w_{i, k}^{2}+\varphi_{i, k}^{2} y_{i, k}^{2}\left(-\frac{2 \lambda_{i} \mu_{i}}{m_{i}^{2}}+1\right) \\
&+2 \varphi_{i, k} y_{i, k} e_{i, k}\left[a_{i 0}+\frac{1}{m_{i}^{2}}\left(-\lambda_{i} a_{i 0}+\lambda_{i} \mu_{i} \theta_{i, k}\right)\right]+\frac{2}{m_{i}^{2}} \lambda_{i} \mu_{i} \theta_{i, k} \varphi_{i, k} y_{i, k} y_{i r}+\frac{2}{m_{i}^{2}}\left(\theta_{i, k}-m_{i} \theta_{i, k-1}\right) \varphi_{i, k} . \\
& S_{i 1}= a_{i 0}+\frac{1}{m_{i}^{2}}\left(-\lambda_{i} a_{i 0}+\lambda_{i} \mu_{i} \theta_{i, k}\right), \\
& S_{i 2}=\frac{1}{m_{i}^{2}} \lambda_{i} \mu_{i} \theta_{i, k} y_{i r}, S_{i 11}=a_{i 0}+\frac{1}{m_{i}^{2}}\left(\lambda_{i} \mu_{i} \delta_{i 1}-\lambda_{i} a_{i 0}\right), \\
& S_{i 3}=\frac{1}{m_{i}^{2}}\left(\theta_{i, k}-m_{i} \theta_{i, k-1}\right) . S_{i 12}=a_{i 0}+\frac{1}{m_{i}^{2}}\left(\lambda_{i} \mu_{i} \delta_{i 2}-\lambda_{i} a_{i 0}\right), \\
& S_{i 31}=\frac{1}{m_{i}^{2}}\left(\delta_{i 1}-m_{i} \delta_{i 2}\right), \\
& S_{i 32}=\frac{1}{m_{i}^{2}}\left(\delta_{i 2}-m_{i} \delta_{i 1}\right) .
\end{aligned}
\end{aligned}
$$

Then, (37) can be rewritten as

$$
\begin{aligned}
\Delta V_{i, k}= & \left(-1+a_{i 0}^{2}\right) e_{i, k}^{2}+\left(-\frac{1}{m_{i}^{2}}+1\right) \varphi_{i, k}^{2}-\frac{1}{m_{i}^{2}} w_{i, k}^{2} \\
& +\varphi_{i, k}^{2} y_{i, k}^{2}\left(-\frac{2 \lambda_{i} \mu_{i}}{m_{i}^{2}}+1\right)+2 S_{i 1} \varphi_{i, k} y_{i, k} e_{i, k} \\
& +2 S_{i 2} \varphi_{i, k} y_{i, k}+2 S_{i 3} \varphi_{i, k} .
\end{aligned}
$$

We denote $S_{i 1 m}=\max \left\{\left|S_{i 11}\right|,\left|S_{i 12}\right|\right\}$ and $S_{i 3 m}=$ $\max \left\{\left|S_{i 31}\right|,\left|S_{i 32}\right|\right\}$. Then, $\left|S_{i 1}\right| \leq S_{i 1 m}$ and $\left|S_{i 3}\right| \leq S_{i 3 m}$. From (20), $\left|S_{i 2}\right| \leq S_{i 2 m}=1 / m_{i}^{2} \lambda_{i} \mu_{i}\left(1+\varepsilon_{i}\right) \cdot\left|y_{i r}\right|$.

Now, employing Lemma 2, we obtain

$$
2 S_{i 1} \varphi_{i, k} y_{i, k} e_{i, k} \leq 2 S_{i 1 m}\left|\varphi_{i, k} y_{i, k}\right| \cdot\left|e_{i, k}\right| \leq \frac{S_{i 1 m}}{\gamma_{i 1}} \varphi_{i, k}^{2} y_{i, k}^{2}+\gamma_{i 1} S_{i 1 m} e_{i, k}^{2} \text {, }
$$

$$
\begin{array}{r}
2 S_{i 2} \varphi_{i, k} y_{i, k} \leq \frac{S_{i 2 m}}{\gamma_{i 2}} \varphi_{i, k}^{2} y_{i, k}^{2}+\gamma_{i 2} S_{i 2 m}, \\
2 S_{i 3} \varphi_{i, k} \leq \frac{S_{i 3 m}}{\gamma_{i 3}} \varphi_{i, k}^{2}+\gamma_{i 3} S_{i 3 m},
\end{array}
$$

For further analysis, we derive the ranges of $S_{i 1}, S_{i 2}$, and $S_{i 3}$ shown in (38). Let $S_{i j 1}$ and $S_{i j 2}$ denote the minimum and maximum values of $S_{i j}(j=1,2,3)$, respectively. Based on the expressions of $S_{i 1}$ and $S_{i 3}$, we have

provided that $\gamma_{i 1}, \gamma_{i 2}$, and $\gamma_{i 3}>0$. Substituting these three inequalities into (39) yields 


$$
\begin{aligned}
\Delta V_{i, k} \leq & \left(-1+a_{i 0}^{2}\right) e_{i, k}^{2}+\left(-\frac{1}{m_{i}^{2}}+1\right) \varphi_{i, k}^{2}-\frac{1}{m_{i}^{2}} w_{i, k}^{2}+\varphi_{i, k}^{2} y_{i, k}^{2}\left(-\frac{2 \lambda_{i} \mu_{i}}{m_{i}^{2}}+1\right)+\frac{S_{i 1 m}}{\gamma_{i 1}} \varphi_{i . k}^{2} y_{i, k}^{2}+\gamma_{i 1} S_{i 1 m} e_{i, k}^{2}+\frac{S_{i 2 m}}{\gamma_{i 2}} \varphi_{i . k}^{2} y_{i, k}^{2} \\
& +\gamma_{i 2} S_{i 2 m}+\frac{S_{i 3 m}}{\gamma_{i 3}} \varphi_{i, k}^{2}+\gamma_{i 3} S_{i 3 m} \\
\leq & \left(-1+a_{i 0}^{2}+\gamma_{i 1} S_{i 1 m}\right) e_{i, k}^{2}+\left(-\frac{2 \lambda_{i} \mu_{i}}{m_{i}^{2}}+1+\frac{S_{i 1 m}}{\gamma_{i 1}}+\frac{S_{i 2 m}}{\gamma_{i 2}}\right) \varphi_{i . k}^{2} y_{i, k}^{2}+\left(-\frac{1}{m_{i}^{2}}+1+\frac{S_{i 3 m}}{\gamma_{i 3}}\right) \varphi_{i, k}^{2}+\gamma_{i 2} S_{i 2 m}+\gamma_{i 3} S_{i 3 m} .
\end{aligned}
$$

Denote

$$
\begin{aligned}
& N_{i 1}=1-a_{i 0}^{2}-\gamma_{i 1} S_{i 1 m}, \\
& N_{i 2}=\frac{2 \lambda_{i} \mu_{i}}{m_{i}^{2}}-1-\frac{S_{i 1 m}}{\gamma_{i 1}}-\frac{S_{i 2 m}}{\gamma_{i 2}}, \\
& N_{i 3}=\frac{1}{m_{i}^{2}}-1-\frac{S_{i 3 m}}{\gamma_{i 3}} \\
& \delta_{i m}=\gamma_{i 2} S_{i 2 m}+\gamma_{i 3} S_{i 3 m} .
\end{aligned}
$$

Then, we have

$$
\Delta V_{i, k} \leq-N_{i 1} e_{i, k}^{2}-N_{i 2} \varphi_{i, k}^{2} y_{i, k}^{2}-N_{i 3} \varphi_{i, k}^{2}+\delta_{i m},
$$

this is,

$$
\begin{aligned}
\Delta V_{i, k}= & e_{i, k+1}^{2}+\varphi_{i, k}^{2}-e_{i, k}^{2}-\varphi_{i, k-1}^{2} \leq-N_{i 1} e_{i, k}^{2} \\
& -N_{i 2} \varphi_{i, k}^{2} y_{i, k}^{2}-N_{i 3} \varphi_{i, k}^{2}+\delta_{i m} .
\end{aligned}
$$

Part 3. Giving the properties of $N_{i 1}, N_{i 2}$, and $N_{i 3}$ in (46).

Based on the bounds of the characteristic parameters provided by (20), we can prove that there exist $\gamma_{i 1}, \gamma_{i 2}$, and $\gamma_{i 3}>0$, such that $N_{i 1} \in(0,1) ; N_{i 2}$ and $N_{i 3}>0$. To prove these, it suffices to show that

$$
2 \lambda_{i} \mu_{i}-m_{i}^{2}>0, \quad \frac{m_{i}^{2} S_{i 1 m}}{2 \lambda_{i} \mu_{i}-m_{i}^{2}}<\frac{1-a_{i 0}^{2}}{S_{i 1 m}} .
$$

Indeed, if (47) holds, then there exist $\gamma_{i 1}$ that satisfy

$$
0<\frac{m_{i}^{2} S_{i 1 m}}{2 \lambda_{i} \mu_{i}-m_{i}^{2}}<\gamma_{i 1}<\frac{1-a_{i 0}^{2}}{S_{i 1 m}} .
$$

Further, we have $N_{i 1}=1-a_{i 0}^{2}-\gamma_{i 1} S_{i 1 m} \in(0,1)$, and $2 \lambda_{i} \mu_{i} \gamma_{i 1}-m_{i}^{2} \gamma_{i 1}-m_{i}^{2} S_{i 1 m}>0$. Thus, there exists a positive number

$$
\gamma_{i 2}>\frac{m_{i}^{2} \gamma_{i 1} S_{i 2 m}}{2 \lambda_{i} \mu_{i} \gamma_{i 1}-m_{i}^{2} \gamma_{i 1}-m_{i}^{2} S_{i 1 m}} .
$$

Note that

$$
N_{i 2}=\frac{2 \lambda_{i} \mu_{i} \gamma_{i 1}-m_{i}^{2} \gamma_{i 1}-m_{i}^{2} S_{1 m}}{m_{i}^{2} \gamma_{i 1}}-\frac{S_{i 2 m}}{\gamma_{i 2}} .
$$

Consequently, we can obtain $N_{i 2}>0$ for any $\gamma_{i 1}$ and $\gamma_{i 2}$ that satisfy (48) and (49), respectively.

In what follows, we shall prove that (47) holds indeed.

For simplicity, the subscript $i$ of variables is omitted until the end of the proof of (47), except for $S_{i 11}$ and $S_{i 12}$. For example, we use the notation $\mu$ instead of $\mu_{i}$.

(i) First, we prove the first formula in (47).

Since

$$
\begin{aligned}
\left\{2 \mu \Delta-c^{2}\right\}^{2}-4 \mu^{2}\left[\Delta^{2}-c^{2}\right] & =4 \mu^{2} \Delta^{2}+c^{4}-4 \mu \Delta c^{2}-4 \mu^{2} \Delta^{2}+4 \mu^{2} c^{2} \\
& =c^{4}-4 \mu \Delta c^{2}+4 \mu^{2} c^{2}=c^{4}-4 \mu\left[-a_{0} c+\left(1-a_{0}^{2}\right) \mu\right] c^{2}+4 \mu^{2} c^{2} \\
& =c^{4}+4 \mu a_{0} c^{3}-4 \mu^{2} c^{2}+4 \mu^{2} a_{0}^{2} c^{2}+4 \mu^{2} c^{2} \\
& >0 .
\end{aligned}
$$

We have $2 \mu \Delta-c^{2}>2 \mu \sqrt{\Delta^{2}-c^{2}}$. Consequently, we obtain $2 \mu\left(\left(\Delta-\sqrt{\Delta^{2}-c^{2}}\right) / c^{2}\right)>1$. Multiplying both sides of the above inequality by $m^{2}$ yields $\left(2 \mu\left(m^{2} \Delta-m^{2} \sqrt{\Delta^{2}-c^{2}}\right) / c^{2}\right)>m^{2}$. That is, $2 \lambda_{1} \mu-$ $m^{2}>0$. Thus, $2 \lambda \mu-m^{2}>0$.

(ii) Next, we prove the second formula in (47).

Note that $\delta_{1}=1-\varepsilon>0$ when the sampling period $T_{s}<T_{\min }$. Since $\mu>a_{0} / \delta_{1}$, we have $\mu \delta_{1}>a_{0}$ and $\mu \delta_{1}-a_{0}>0$. Therefore, $S_{i 11}>0$. Thus, we obtain $S_{1 m}=S_{i 12}=a_{0}+1 / m^{2}\left(\lambda \mu \delta_{2}-\lambda a_{0}\right)$. Then, from $c=\mu \delta_{2}-a_{0}$, we have

$$
\begin{aligned}
m^{2} S_{1 m}^{2}-\left(1-a_{0}^{2}\right)\left(2 \lambda \mu-m^{2}\right)= & \frac{1}{m^{2}}\left[c \lambda+m^{2} a_{0}\right]^{2} \\
& -\left(1-a_{0}^{2}\right)\left(2 \lambda \mu-m^{2}\right) \\
= & \frac{1}{m^{2}}\left[c^{2} \lambda^{2}-2\left[-m^{2} a_{0} c\right.\right. \\
& \left.\left.+m^{2}\left(1-a_{0}^{2}\right) \mu\right] \lambda+m^{4}\right] .
\end{aligned}
$$


From $\lambda \in\left(\lambda_{1}, \lambda_{2}\right)$, we have $c^{2} \lambda^{2}-2\left[-m^{2} a_{0} c+\right.$ $\left.m^{2}\left(1-a_{0}^{2}\right) \mu\right] \lambda+m^{4}<0$, i.e.,

$$
m^{2} S_{1 m}^{2}<\left(1-a_{0}^{2}\right)\left(2 \lambda \mu-m^{2}\right)
$$

Therefore, we have $m^{2} S_{1 m} /\left(2 \lambda \mu-m^{2}\right)<\left(1-a_{0}^{2}\right) / S_{1 m}$. Thus, the second formula in (47) is proved.

Now, we notice that $N_{i 3}>0$ can be guaranteed by taking positive numbers $\gamma_{i 3}>m_{i}^{2} S_{i 3 m} /\left(1-m_{i}^{2}\right)$.
Part 4. Proving the boundedness for the tracking errors $e_{i, k}$ and parameter estimation error $\varphi_{i, k}$ and convergence for tracking errors $e_{i, k}$.

From the above results and (46), we can obtain the following result:

$$
\begin{aligned}
e_{i, k+1}^{2}+\left(1+N_{i 3}\right) \varphi_{i, k}^{2} & \leq\left(1-N_{i 1}\right) e_{i, k}^{2}+\varphi_{i, k-1}^{2}-N_{i 2} \varphi_{i, k}^{2} y_{i, k}^{2}+\delta_{i m} \\
& \leq\left(1-N_{i 1}\right) e_{i, k}^{2}+\varphi_{i, k-1}^{2}+\delta_{i m} .
\end{aligned}
$$

Denote $\quad N_{i}=\max \left\{1 /\left(1+N_{i 3}\right), 1-N_{i 1}\right\} ; \quad$ then, $N_{i} \in(0,1)$. Thus, we have

$$
e_{i, k+1}^{2}+\left(1+N_{i 3}\right) \varphi_{i, k}^{2} \leq\left(1-N_{i 1}\right) e_{i, k}^{2}+\frac{1}{1+N_{i 3}}\left[\left(1+N_{i 3}\right) \varphi_{i, k-1}^{2}\right]+\delta_{i m} \leq N_{i}\left[e_{i, k}^{2}+\left(1+N_{i 3}\right) \varphi_{i, k-1}^{2}\right]+\delta_{i m}
$$

In other words, we obtained a recursion relation that can yield

$$
\begin{aligned}
e_{i, k+1}^{2}+\left(1+N_{i 3}\right) \varphi_{i, k}^{2} & \leq N_{i}^{2}\left[e_{i, k-1}^{2}+\left(1+N_{i 3}\right) \varphi_{i, k-2}^{2}\right]+N_{i} \delta_{i m}+\delta_{i m} \leq \cdots \\
& \leq N_{i}^{k}\left[e_{i, 1}^{2}+\left(1+N_{i 3}\right) \varphi_{i, 0}^{2}\right]+N_{i}^{k-1} \delta_{i m}+N_{i}^{k-2} \delta_{i m}+\cdots+N_{i}^{2} \delta_{i m}+N_{i} \delta_{i m}+\delta_{i m} \\
& =N_{i}^{k}\left[e_{i, 1}^{2}+\left(1+N_{i 3}\right) \varphi_{i, 0}^{2}\right]+\frac{1-N_{i}^{k}}{1-N_{i}} \delta_{i m} \\
& <e_{i, 1}^{2}+\left(1+N_{i 3}\right) \varphi_{i, 0}^{2}+\frac{1}{1-N_{i}} \delta_{i m} .
\end{aligned}
$$

From this inequality, both $e_{i, k}$ and $\varphi_{i, k}$ are bounded, and we can obtain

$$
e_{i, k+1}^{2} \leq N_{i}^{k}\left[e_{i, 1}^{2}+\left(1+N_{i 3}\right) \varphi_{i, 0}^{2}\right]+\frac{1-N_{i}^{k}}{1-N_{i}} \delta_{i m},
$$

which implies $\limsup _{k \longrightarrow+\infty} e_{i, k+1}^{2} \leq \delta_{i m} /\left(1-N_{i}\right)$. Thus, $\limsup _{k \rightarrow+\infty}\left|e_{i, k}\right| \leq \sqrt{\delta_{i m} /\left(1-N_{i}\right)}$. By the definition of the supremum limit, there exist integers $M_{i}>0$ for all sufficiently small $\bar{\varepsilon}_{i}>0$ such that

$$
\left|e_{i, k}\right| \leq \rho_{i}=\max \left\{\left|e_{i, 1}\right|, \ldots,\left|e_{i, M_{i}}\right|, \bar{\varepsilon}_{i}+\sqrt{\frac{\delta_{i m}}{1-N_{i}}}\right\} \text {. }
$$

Hence, Theorem 2 is proved.

Remark 2. If $f_{i}(x, u)$ is bounded, that is, there exists a constant $L_{i}>0$ such that $\left|f_{i}(x, u)\right| \leq L_{i}$, then the tracking error $e_{i}(t)=x_{i}(t)-x_{i r}$ of system (1) converges to the neighborhood of the origin under the conditions in Theorem 2. In fact, for any $t \in\left[k T_{s},(k+1) T_{s}\right]$, integrating (1) from $k T_{s}$ to $t$ and noting $\dot{e}_{i}(t)=\dot{x}_{i}(t)$, one can easily obtain $\left|e_{i}(t)\right| \leq\left|e_{i}\left(k T_{s}\right)\right|+T_{s} L_{i} \leq \rho_{i}+T_{s} L_{i}$, where $\rho_{i}$ is defined in (58).

\section{Numerical Example}

To verify the effectiveness of the developed control method, consider a nonaffine double-pendulum system. The dynamic equations of motion are given as [44]

$$
\begin{aligned}
& {\left[\begin{array}{c}
\dot{x}_{1} \\
\dot{x}_{2} \\
\dot{x}_{3} \\
\dot{x}_{4}
\end{array}\right]=\left[\begin{array}{llll}
0 & 1 & 0 & 0 \\
0 & 0 & 0 & 0 \\
0 & 0 & 0 & 1 \\
0 & 0 & 0 & 0
\end{array}\right]\left[\begin{array}{l}
x_{1} \\
x_{2} \\
x_{3} \\
x_{4}
\end{array}\right]+\left[\begin{array}{ll}
0 & 0 \\
1 & 0 \\
0 & 0 \\
0 & 1
\end{array}\right]\left[\begin{array}{l}
f_{1}(x, u) \\
f_{2}(x, u)
\end{array}\right],} \\
& y(t)=\left[\begin{array}{llll}
1 & 0 & 0 & 0 \\
0 & 0 & 1 & 0
\end{array}\right] x(t),
\end{aligned}
$$

with $x(0)=[0.2,0.1,0.2,0.1]^{\mathrm{T}} . x=\left[x_{1}, x_{2}, x_{3}, x_{4}\right]^{\mathrm{T}}$ is the vector of measurable states, $u=\left[u_{1} u_{2}\right]^{\mathrm{T}}$ is the vector control inputs, and

$$
\begin{aligned}
& f_{1}(x, u)=f_{11}(x) M_{1}\left(x, u_{1}\right)+f_{12}(x) M_{2}\left(x, u_{2}\right)+f_{13}(x), \\
& f_{2}(x, u)=f_{21}(x) M_{1}\left(x, u_{1}\right)+f_{22}(x) M_{2}\left(x, u_{2}\right)+f_{23}(x) .
\end{aligned}
$$


Here,

$$
\begin{aligned}
f_{11}\left(x_{1}, x_{3}\right)= & \frac{12}{l_{1}^{2}\left[4 m_{1}+12 m_{2}-9 m_{2} \cos ^{2}\left(x_{3}-x_{1}\right)\right]}, \\
f_{12}\left(x_{1}, x_{3}\right)= & \frac{12 l_{2}+18 l_{1} \cos \left(x_{3}-x_{1}\right)}{l_{1}^{2} l_{2}\left[9 m_{2} \cos ^{2}\left(x_{3}-x_{1}\right)-4 m_{1}-12 m_{2}\right]}, \\
f_{21}\left(x_{1}, x_{3}\right)= & \frac{18 \cos \left(x_{3}-x_{1}\right)}{l_{1} l_{2}\left[9 m_{2} \cos ^{2}\left(x_{3}-x_{1}\right)-4 m_{1}-12 m_{2}\right]}, \\
f_{22}\left(x_{1}, x_{3}\right)= & \frac{12 m_{1}+36 m_{2}}{l_{2}^{2} m_{2}\left[4 m_{1}+12 m_{2}-9 m_{2} \cos ^{2}\left(x_{3}-x_{1}\right)\right]}+\frac{18 l_{2} \cos \left(x_{3}-x_{1}\right)}{l_{1} l_{2}^{2}\left[4 m_{1}+12 m_{2}-9 m_{2} \cos ^{2}\left(x_{3}-x_{1}\right)\right]}, \\
f_{13}\left(x_{1}, x_{2}, x_{3}, x_{4}\right)= & \frac{9 g m_{2} \sin \left(2 x_{3}-x_{1}\right)}{l_{1}\left[15 m_{2}+8 m_{1}-9 m_{2} \cos \left(2 x_{3}-2 x_{1}\right)\right]}-\frac{9 l_{1} m_{2} x_{2}^{2} \sin \left(2 x_{3}-2 x_{1}\right)}{l_{1}\left[15 m_{2}+8 m_{1}-9 m_{2} \cos \left(2 x_{3}-2 x_{1}\right)\right]} \\
& +\frac{12 l_{2} m_{2} x_{4}^{2} \sin \left(x_{3}-x_{1}\right)}{l_{1}\left[15 m_{2}+8 m_{1}-9 m_{2} \cos \left(2 x_{3}-2 x_{1}\right)\right]}-\frac{\left(15 g m_{2}+12 g m_{1}\right) \sin \left(x_{1}\right)}{l_{1}\left[15 m_{2}+8 m_{1}-9 m_{2} \cos \left(2 x_{3}-2 x_{1}\right)\right]}, \\
f_{23}\left(x_{1}, x_{2}, x_{3}, x_{4}\right)= & \frac{12 l_{1}^{2} x_{2}^{2} \sin \left(x_{3}-x_{1}\right)\left(m_{1}+3 m_{2}\right)}{l_{1}\left[15 m_{2}+8 m_{1}-9 m_{2} \cos \left(2 x_{3}-2 x_{1}\right)\right]}-\frac{9 l_{2} m_{2} x_{4}^{2} \sin \left(2 x_{3}-2 x_{1}\right)}{l_{1}\left[15 m_{2}+8 m_{1}-9 m_{2} \cos \left(2 x_{3}-2 x_{1}\right)\right]} \\
& -\frac{9 g \sin \left(x_{3}-2 x_{1}\right)\left(m_{1}+2 m_{2}\right)}{l_{1}\left[15 m_{2}+8 m_{1}-9 m_{2} \cos \left(2 x_{3}-2 x_{1}\right)\right]}+\frac{3 g \sin \left(x_{3}\right)\left(m_{1}+6 m_{2}\right)}{l_{1}\left[15 m_{2}+8 m_{1}-9 m_{2} \cos \left(2 x_{3}-2 x_{1}\right)\right]} ; \\
M_{1}\left(u_{1}\right)= & \tanh \left(u_{1}\right)+0.35 u_{1}, \\
M_{2}\left(u_{2}\right)= & \tanh \left(u_{2}\right)+0.35 u_{2},
\end{aligned}
$$

where $m_{1}$ and $m_{2}$ are the mass of pendulums 1 and 2, respectively, and the meaning of other symbols in (61) can be found in [44].

Herein, the control objective is to use our adaptive controller such that the states $x_{1}$ and $x_{3}$ are adjusted to 0 from the initial state. In this example, the parameter values of the nonaffine double-pendulum system are $m_{1}=m_{2}=$ $1 \mathrm{~kg}, l_{1}=l_{2}=1 \mathrm{~m}$, and $g=9.81 \mathrm{~m} / \mathrm{s}^{2}$. The initial values of the parameters $\widehat{\theta}_{i, k}$ are chosen to be $\widehat{\theta}_{i, 0}=1$ for each $i=1,2$. The parameters of the controller are selected as $a_{i 0}=0.01 T_{s}$, $\lambda_{i}=1000, \mu_{i}=0.001$, and $m_{i}=0.1 T_{s}$ for $i=1,2$. Meanwhile, we set $\eta_{i}=0.0001, d_{i}=0.0002$ for $i=1,2$, and the sampling period $T_{s}=0.001$.

Figures 1-4 depict the simulation results. As shown in the figures, the system can effectively track the expected outputs, thereby confirming the effectiveness of our proposed control approach.

To demonstrate the effectiveness of the proposed control strategy, we use the fuzzy adaptive control method in [45] instead of our proposed adaptive controller. The control strategy proposed in [45] can be described as follows.

Control laws are designed as follows:

$$
u_{i}=u_{d i}+u_{f i}+u_{h i}, \quad i=1,2,
$$

where $u_{d i}=K_{i}^{\mathrm{T}} \bar{e}_{i}, \bar{e}_{i}=\left[e_{i}, \dot{e}_{i}\right]^{\mathrm{T}}, e_{i}$ is the tracking error of $x_{i}$, $K_{i}=\left[k_{i 2}, k_{i 1}\right]^{\mathrm{T}}$, and all roots of the polynomial $h(s)=s^{2}+$ $k_{i 1} s+k_{i 2}$ are in the open left-half plane; the fuzzy control terms $u_{f i}=\theta_{i}^{\mathrm{T}} \xi_{i}(x)$, in which $\xi_{i}(x)$ is fuzzy basis function, and we use the following adaptation laws to adjust the unknown parameter vectors of fuzzy systems:

$$
\dot{\theta}_{i}=-\eta_{i} \bar{e}_{i}^{T} P_{i} B \xi_{i}(x)-\sigma_{i} \eta_{i}\left\|\bar{e}_{i}\right\| \theta_{i},
$$

where $\eta_{i}, \sigma_{i}>0$ are adaptive parameter rates;

$$
u_{h i}=-\frac{1}{r_{i}} B^{\mathrm{T}} P_{i} e_{i}, \quad i=1,2,
$$

where $r_{i}>0$ is a design parameter, $B=[0,1]^{\mathrm{T}}$, and $P_{i}$ is a symmetric positive definite matrix satisfying the Riccati equation

$$
A_{i}^{T} P_{i}+P_{i} A_{i}+Q_{i}-\left(\frac{2}{r_{i}}-\frac{1}{\rho_{i}^{2}}\right) P_{i} B B^{T} P_{i}=0,
$$

where $Q_{i}>0$ and $A_{i}=\left[\begin{array}{cc}0 & 1 \\ -k_{i 2} & -k_{i 1}\end{array}\right]$.

In the simulation, the design parameters are $k_{i 1}=2$, $k_{i 2}=1, \quad Q_{i}=\left[\begin{array}{cc}100 & 0 \\ 0 & 100\end{array}\right], \quad r_{i}=0.001$, and $\rho_{i}=0.1$, for 


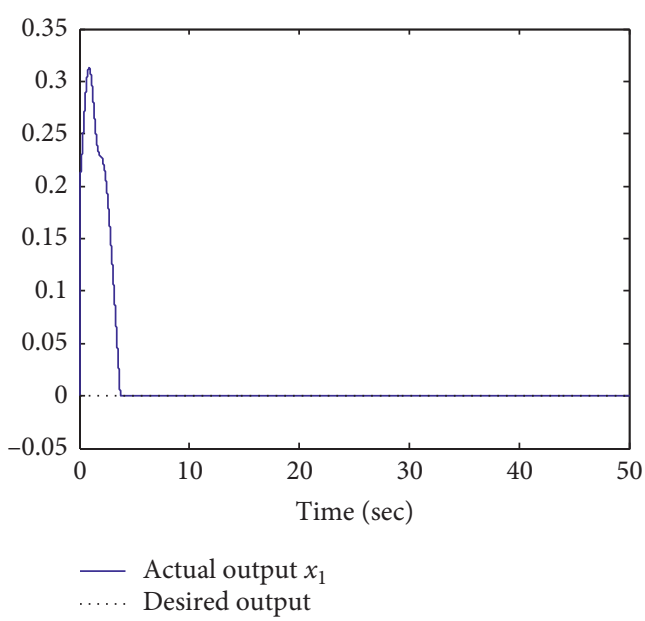

FIgURE 1: Trajectories of the first output $x_{1}$ for our controller.

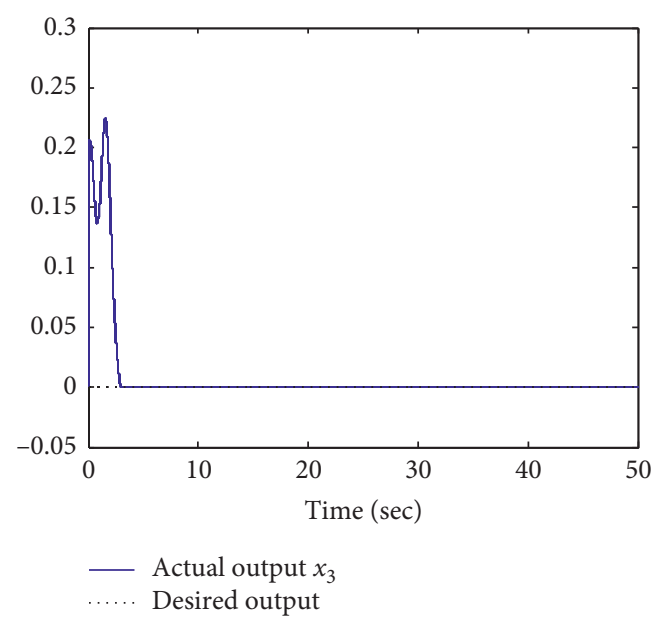

Figure 2: Trajectories of the second output $x_{3}$ for our controller.

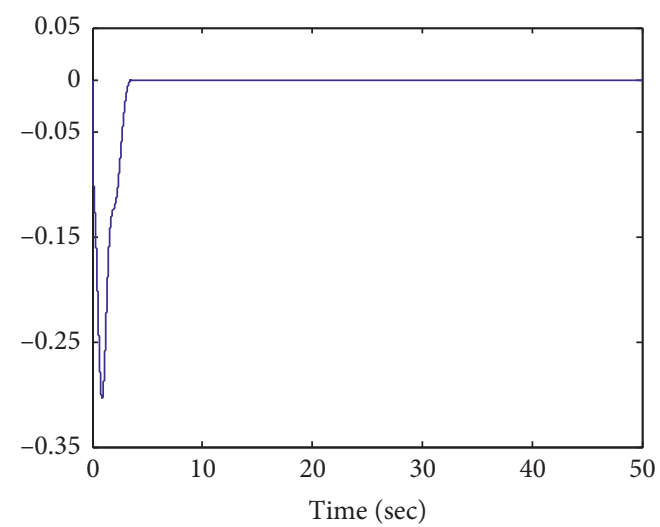

- Control input $u_{1}$

Figure 3: Control input $u_{1}$ for our controller.

$i=1,2$. The adaptive parameter rates are chosen as $\eta_{i}=0.1$ and $\sigma_{i}=10$, for $i=1,2$. With respect to fuzzy logic systems, Mamdani fuzzy models are employed to obtain the fuzzy

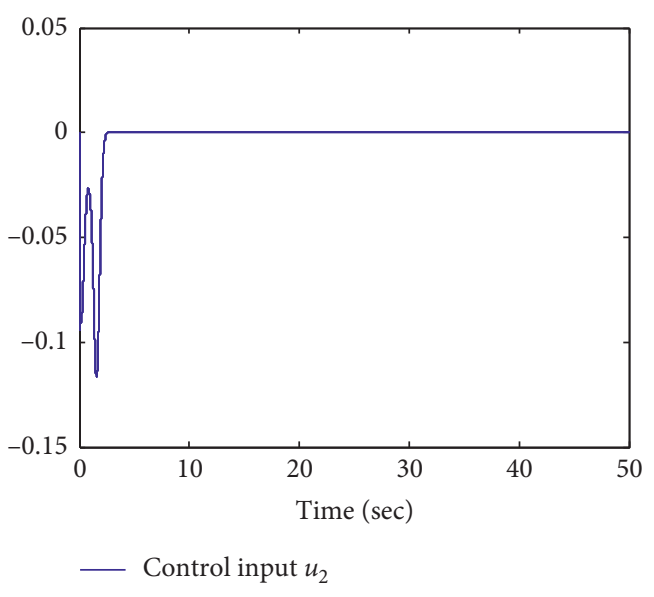

FIGURE 4: Control input $u_{2}$ for our controller.

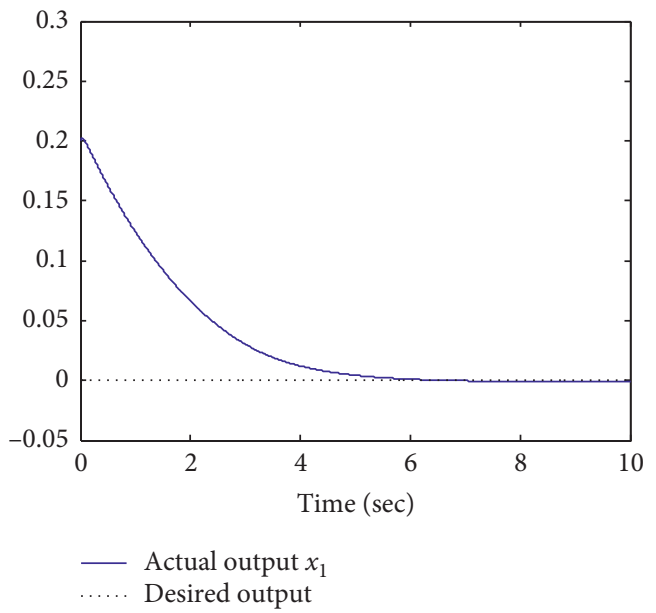

FIgURE 5: Trajectories of the first output $x_{1}$ for the fuzzy controller [45].

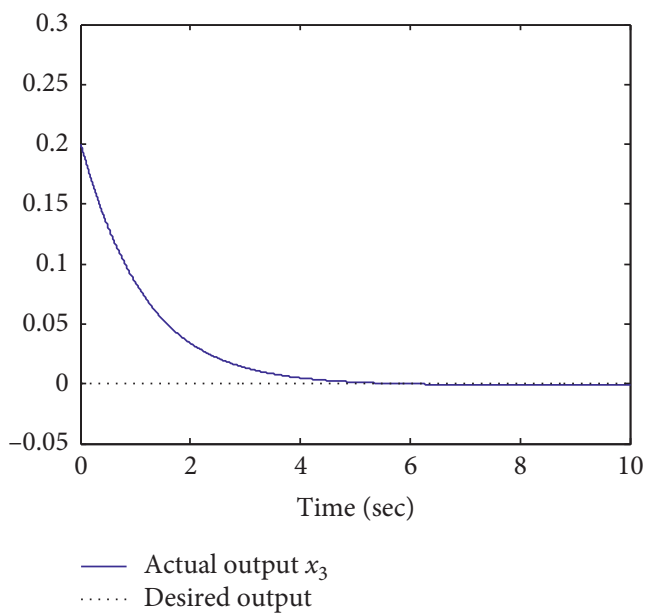

Figure 6: Trajectories of the second output $x_{3}$ for the fuzzy controller [45].

control terms $u_{f 1}$ and $u_{f 2}$, and we define five Gaussian membership functions for each variable $x_{i}, i=1,2,3,4$, distributed uniformly on interval $[-0.8,0.8]$. Then, there are 


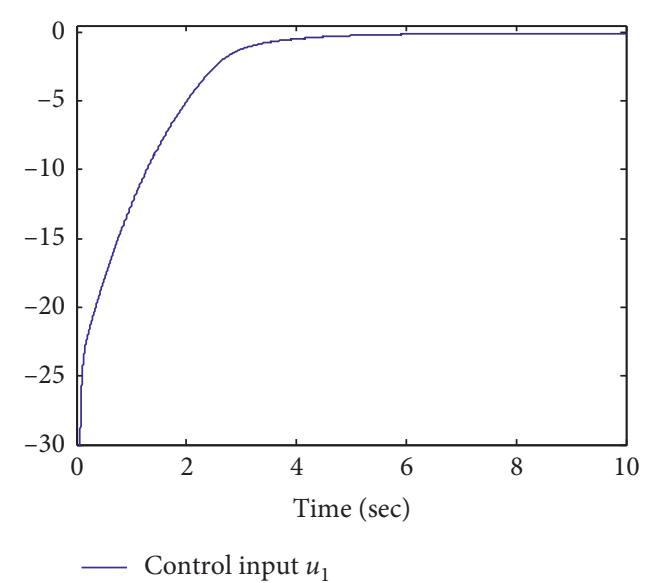

FIGURE 7: Control input $u_{1}$ for the fuzzy controller [45].

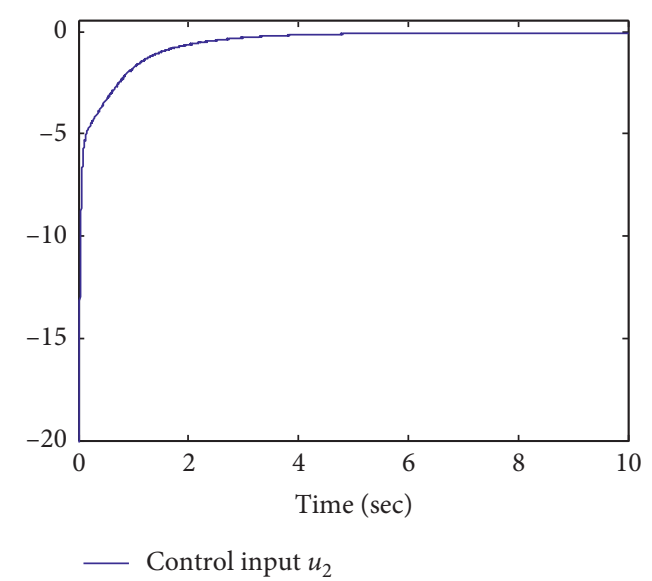

Figure 8: Control input $u_{2}$ for the fuzzy controller [45].

$2 \times 5 \times 5 \times 5 \times 5=1250$ rules that are used in the simulation. The initial state of each variable is the same as in the above simulation.

The results of this simulation are illustrated in Figures 5-8. The figures indicate that the adaptive fuzzy controller can also achieve better performance. However, it should be emphasized that this is the result of more than 2 min of CPU time running on a computer (Lenovo workstation P720 with dual Intel ${ }^{\circledR}$ Xeon $^{\circledR}$ Gold 6136 Processor), whereas the results shown in Figures 1-4 only require $7 \mathrm{~s}$ of CPU time on the same computer.

There are 1250 parameters that need to be updated online for the scheme in [45], whereas only 2 parameters are required in our scheme. The fewer parameters used in our scheme help to make the proposed algorithm more efficient.

\section{Conclusions}

In this study, the control method for a class of uncertain nonaffine systems is studied for the first time by using a firstorder characteristic model. Specifically, we first transformed the nonaffine uncertain system into a linear first-order characteristic model with bounded time-varying parameters. Based on this model, we designed an adaptive controller with a simple structure and proved that the tracking errors converge to the neighborhoods of the origin. The proposed approach can avoid the controller singularity because there is no inverse operator construction in the controller. As an illustration of the effectiveness of the proposed method, a numerical example is provided.

\section{Data Availability}

No data were used to support this study.

\section{Conflicts of Interest}

The authors declare that there are no conflicts of interest regarding the publication of this paper.

\section{Acknowledgments}

This work was supported by the National Nature Science Foundation of China (no. 61333008) and the Scientific Research Fund of Hebei Normal University of Science and Technology (no. 2018HY021).

\section{References}

[1] C. J. Silva, H. Maurer, and D. F. M. Torres, "Optimal control of a tuberculosis model with state and control delays," Mathematical Biosciences and Engineering, vol. 14, no. 1, pp. 321337, 2017.

[2] Y. Li, X. Xu, and W. Wang, "GA-BPNN based hybrid steering control approach for unmanned driving electric vehicle with in-wheel motors," Complexity, vol. 2018, Article ID 6132139, 15 pages, 2018.

[3] X. Liu and P. Stechlinski, "Switching and impulsive control algorithms for nonlinear hybrid dynamical systems," Nonlinear Analysis: Hybrid Systems, vol. 27, pp. 307-322, 2018.

[4] P. Niamsup, K. Ratchagit, and V. N. Phat, "Novel criteria for finite-time stabilization and guaranteed cost control of delayed neural networks," Neurocomputing, vol. 160, pp. 281-286, 2015.

[5] B. Yi, S. Lin, B. Yang, and W. Zhang, "Performance recovery of a class of uncertain non-affine systems with unmodelled dynamics: an indirect dynamic inversion method," International Journal of Control, vol. 91, no. 2, pp. 266-284, 2018.

[6] B. Kiumarsi, W. Kang, and F. L. Lewis, "Ho control of nonaffine aerial systems using off-policy reinforcement learning," Unmanned Systems, vol. 04, no. 01, pp. 51-60, 2016.

[7] Y.-H. Liu, L. Huang, and D. Xiao, "Adaptive dynamic surface control for uncertain nonaffine nonlinear systems," International Journal of Robust and Nonlinear Control, vol. 27, no. 4, pp. 535-546, 2017.

[8] P. Kumar, N. Kumar, and V. Panwar, "RBF neural control design for SISO nonaffine nonlinear systems," Procedia Computer Science, vol. 125, pp. 25-33, 2018.

[9] R. Choe, E. Xargay, and N. Hovakimyan, " $L_{1}$ adaptive control for a class of uncertain nonaffine-in-control nonlinear systems," IEEE Transactions on Automatic Control, vol. 61, no. 3, pp. 840-846, 2016.

[10] W. Meng, Q. Yang, S. Jagannathan, and Y. Sun, "Adaptive neural control of high-order uncertain nonaffine systems: a transformation to affine systems approach," Automatica, vol. 50, no. 5, pp. 1473-1480, 2014. 
[11] Z. Zhen, S. Jiang, and K. Ma, "Automatic carrier landing control for unmanned aerial vehicles based on preview control and particle filtering," Aerospace Science and Technology, vol. 81, pp. 99-107, 2018.

[12] M. Ran, Q. Wang, and C. Dong, "Active disturbance rejection control for uncertain nonaffine-in-control nonlinear systems," IEEE Transactions on Automatic Control, vol. 62, no. 11, pp. 5830-5836, 2017.

[13] F. Zouari, A. Ibeas, A. Boulkroune, J. Cao, and M. Mehdi Arefi, "Adaptive neural output-feedback control for nonstrictfeedback time-delay fractional-order systems with output constraints and actuator nonlinearities," Neural Networks, vol. 105, pp. 256-276, 2018.

[14] M. Chen, H. Wang, X. Liu, T. Hayat, and F. E. Alsaadi, "Adaptive finite-time dynamic surface tracking control of nonaffine nonlinear systems with dead zone," Neurocomputing, vol. 366, pp. 66-73, 2019.

[15] Q. Lin, R. Loxton, and K. Lay Teo, "The control parameterization method for nonlinear optimal control: a survey," Journal of Industrial \& Management Optimization, vol. 10, no. 1, pp. 275-309, 2014.

[16] C. P. Bechlioulis and G. A. Rovithakis, "Robust adaptive fuzzy control of nonaffine systems guaranteeing transient and steady state error bounds," International Journal of Adaptive Control and Signal Processing, vol. 26, no. 7, pp. 576-591, 2012.

[17] W.-D. Zhou, C.-Y. Liao, L. Zheng, and M.-M. Liu, "Adaptive fuzzy output feedback control for a class of nonaffine nonlinear systems with unknown dead-zone input," Nonlinear Dynamics, vol. 79, no. 4, pp. 2609-2621, 2015.

[18] L.-B. Wu and G.-H. Yang, "Adaptive fuzzy tracking control for a class of uncertain nonaffine nonlinear systems with dead-zone inputs," Fuzzy Sets and Systems, vol. 290, pp. 1-21, 2016.

[19] L.-B. Wu, G.-H. Yang, H. Wang, and F. Wang, "Adaptive fuzzy asymptotic tracking control of uncertain nonaffine nonlinear systems with non-symmetric dead-zone nonlinearities," Information Sciences, vol. 348, pp. 1-14, 2016.

[20] A. Molavi, A. Jalali, and M. Ghasemi Naraghi, "Adaptive fuzzy control of a class of nonaffine nonlinear system with input saturation based on passivity theorem," ISA Transactions, vol. 69, pp. 202-213, 2017.

[21] F. G. Hesam and A. A. Kalat, "Observer-based hybrid adaptive fuzzy control for affine and nonaffine uncertain nonlinear systems," Neural Computing \& Applications, vol. 30, no. 4, pp. 1187-1202, 2018.

[22] S. Doudou and F. Khaber, "Adaptive fuzzy sliding mode control for a class of uncertain nonaffine nonlinear strictfeedback systems," Iranian Journal of Science and Technology, Transactions of Electrical Engineering, vol. 43, no. 1, pp. 33-45, 2019.

[23] S.-L. Dai, C. Wang, and M. Wang, "Dynamic learning from adaptive neural network control of a class of nonaffine nonlinear systems," IEEE Transactions on Neural Networks and Learning Systems, vol. 25, no. 1, pp. 111-123, 2014.

[24] T. Sun and Y. Pan, "Adaptive control for nonaffine nonlinear systems using reliable neural network approximation," IEEE Access, vol. 5, pp. 23657-23662, 2017.

[25] X. Wang, X. Li, Q. Wu, and X. Yin, "Neural network based adaptive dynamic surface control of nonaffine nonlinear systems with time delay and input hysteresis nonlinearities," Neurocomputing, vol. 333, pp. 53-63, 2019.
[26] Q. Song and Y. D. Song, "Generalized PI control design for a class of unknown nonaffine systems with sensor and actuator faults," Systems \& Control Letters, vol. 64, pp. 86-95, 2014.

[27] D. Yang and R. Ding, "Transforms from differential equations to difference equations and vice-versa applied to computer control systems," Applied Mathematics Letters, vol. 31, pp. 18-24, 2014.

[28] H. Wu, J. Hu, and Y. Xie, "Characteristic model-based allcoefficient adaptive control method and its applications," IEEE Transactions on Systems, Man and Cybernetics, Part C (Applications and Reviews), vol. 37, no. 2, pp. 213-221, 2007.

[29] D. Q. Sun and Z. M. Sun, "Asymptotic stability of the goldensection control law for multi-input and multi-output linear systems," Journal of Applied Mathematics, vol. 2012, Article ID 407409, 11 pages, 2012.

[30] Y. Wu, Z. Wang, Y. Li, W. Chen, R. Du, and Q. Chen, "Characteristic modeling and control of servo systems with backlash and friction," Mathematical Problems in Engineering, vol. 2014, Article ID 328450, 21 pages, 2014.

[31] H. Huang, "Multiple characteristic model-based golden-section adaptive control: stability and optimization," International Journal of Adaptive Control and Signal Processing, vol. 29, no. 7, pp. 877-904, 2015.

[32] D. Q. Sun, "Stability analysis of golden-section adaptive control systems based on the characteristic model," Science China Information Sciences, vol. 60, no. 9, 18 pages, 2017.

[33] X. J. Lyu, L. Di, Z. L. Lin, Y. F. Hu, and H. C. Wu, "Characteristic model based all-coefficient adaptive control of an AMB suspended energy storage flywheel test rig," Science China Information Sciences, vol. 61, no. 11, 15 pages, 2018.

[34] Y. Wu and G.-P. Liu, "Dual pipeline pressure synchronouscoordinated control with the assistance of the golden section control method," International Journal of Systems Science, vol. 49, no. 11, pp. 2318-2327, 2018.

[35] X. Lyu, L. Di, and Z. Lin, "On robustness of an AMB suspended energy storage flywheel platform under characteristic model based all-coefficient adaptive control laws," Frontiers of Information Technology \& Electronic Engineering, vol. 20, no. 1, pp. 120-130, 2019.

[36] H. Zhong, Y. Wang, H. Ran, Q. Wang, and C. Shao, "Novel distributed PZT active vibration control based on characteristic model for the space frame structure," Shock and Vibration, vol. 2016, Article ID 5928270, 9 pages, 2016.

[37] H. Zhong, Q. Wang, J.-H. Yu, Y.-H. Wei, and Y. Wang, "Discrete sliding mode adaptive vibration control for space frame based on characteristic model," Journal of Computational and Nonlinear Dynamics, vol. 12, no. 1, 8 pages, 2017.

[38] C. Zhou, Y. Shi, S.-H. Yang, Q. Yin, and Y. Qin, "Characteristic model-based adaptive discrete-time sliding mode control for the swing arm in a fourier transform spectrometer," IEEE Transactions on Systems, Man, and Cybernetics, Part C (Applications and Reviews), vol. 42, no. 6, pp. 16331643, 2012.

[39] L.-J. Xu and Y. Hu, "Adaptive tracking control method based on the first-order characteristic models," Control and Decision, vol. 31, no. 9, pp. 1692-1696, 2016.

[40] M. Sun, Z. Li, and L. Yu, "The first-order characteristic models of dynamic systems and adaptive iterative learning control of linear servo system," Journal of Systems Science and Mathematical Sciences, vol. 32, no. 6, pp. 666-682, 2012.

[41] H. X. Wu and Y. Wang, "Characteristic modeling method of the nonlinear system," in Proceedings of the 21st Chinese Control Conference, pp. 138-142, Zhejiang University Press, Hangzhou, Zhejiang, China, 2002. 
[42] W. Shi, "Adaptive fuzzy control for MIMO nonlinear systems with nonsymmetric control gain matrix and unknown control direction," IEEE Transactions on Fuzzy Systems, vol. 22, no. 5, pp. 1288-1300, 2014.

[43] W. Zhang, J. Cao, D. Chen, and A. Alsaedi, "Out lag synchronization of fractional order delayed complex networks with coupling delay via pinning control," Complexity, vol. 2019, Article ID 5612150, 7 pages, 2019.

[44] I.-H. Li and L.-W. Lee, "Interval type 2 hierarchical FNN with the H-infinity condition for MIMO non-affine systems," Applied Soft Computing, vol. 12, no. 8, pp. 1996-2011, 2012.

[45] N.-B. He, Q. Gao, C.-S. Jiang, and C.-L. Gong, "Adaptive fuzzy control for MIMO non-affine nonlinear systems," Control Theory \& Applications, vol. 27, no. 12, pp. 1783-1786, 2010. 